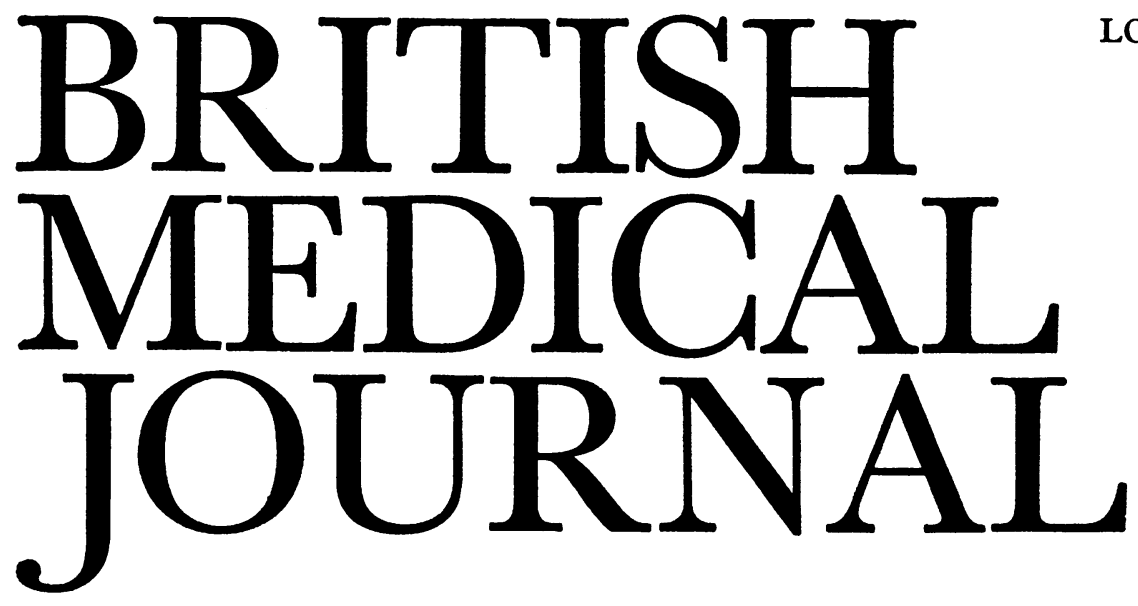

LONDON, SATURDAY 15 FEBRUARY 1975

\title{
Should We Regard Hodgkin's Disease as Infectious?
}

Environmental factors are known to be important in the aetiology of Hodgkin's disease, and recent interest has centred on the possible occurrence of case-to-case transmission, which might suggest an infectious cause.

An extended "epidemic" of cases at a high school in New York State was described by Vianna, Greenwald, and Davies ${ }^{1}$ in 1971. Hodgkin's disease developed in four students who had attended one school at the same time. Subsequent inquiry brought to light 31 additional cases who had had direct contact with the original four or contact through a single intermediary. ${ }^{2}$ The "epidemic" extended over a period of two decades, suggesting a long incubation period. Transmission seemed to occur particularly among young people and from young to the old.

A similar type of outbreak with five cases occurring over a period of 11 years in a small Ohio township containing 405 homes and a population of around 1300 was reported by Klinger and Minton. ${ }^{3}$ The difficulty in interpreting such events has, however, been emphasized by Smith, Pike, and Kinlen $^{4}$ : if the study in a particular area is initiated because a small cluster has already occurred then the population has been artificially selected. The usual tests of statistical significance cannot, therefore, be applied.

As an extension of their previous observations Vianna and Polan $^{5}$ investigated the hypothesis that in schools where an index case of Hodgkin's disease had occurred there would be a subsequent excess of additional cases among students or staff. In five out of eight such secondary schools (with index cases in 1960-64) additional cases were diagnosed during the years 1965-69. In matched control schools there were no cases during the same period, and the difference in incidence was statistically significant. Subsequent correspondence in the columns of the New England Fournal of Medicine has shown questionable support for this finding. Milham ${ }^{6}$ reported a twoto-threefold higher incidence in schoolteachers in the State of Washington. These observations have, however, been criticized on the grounds that the proportional mortality method of analysis fails to take into account that schoolteachers have a lower mortality from other causes and that they belong to higher social classes in which there is a known higher incidence of Hodgkin's disease. ${ }^{7} 8$ Recalculation of Milham's data by Hoover ${ }^{8}$ suggested that the transmission between teachers and students was not a major epidemiological feature of Hodgkin's disease.

Vianna et al. ${ }^{9}$ have more recently asked whether there is an increased mortality from Hodgkin's disease among doctors.
Their survey in upstate New York appeared to show a significantly higher incidence (relative risk 1.8) in doctors than in the general population, dentists, or a population of high economic status; and they suggested that this increase might have been due to contact with patients with Hodgkin's disease. Again, however, this conclusion has been challenged. Smith, Kinlen, and Doll ${ }^{10}$ referred to the incidence of Hodgkin's disease recorded in the prospective study of the mortality of British doctors in relation to their smoking habits. A total of 12 deaths from Hodgkin's disease occurred over a 15-year period compared with an expected 15.48 deaths, giving a relative risk of $0 \cdot 8$. Furthermore the specialty of the 12 doctors dying would have been unlikely to bring them into especially frequent contact with the disease; while among radiotherapists, in whom a high degree of contact would be expected, two small surveys have shown somewhat less than the expected rate. ${ }^{10}$

The most recent contribution from the Albany group of workers ${ }^{11}$ has concerned Hodgkin's disease in the family; they have found that there is about a three-fold increase in incidence among first-degree blood relatives. They confirmed the earlier finding of MacMahon ${ }^{12}$ that the time interval between diagnoses was shorter than the age interval among familial cases, and they also found that the time interval was shorter when the two individuals lived in the same household than when they lived apart. Both those observations favour an environmental rather than a genetic aetiology. The two common familial patterns seen were sib-sib and parent-child; no husband-wife pairs were found-and the authors point out that to their knowledge there have been only five reports of the disease occurring in married couples. Interestingly, the Rye subtypes of the disease were the same among most familial pairs, which may indicate that the pattern of host reactivity, or resistance to progression of the disease, is related to genetic factors. This is supported by the relation between HL-A phenotype and histological classification, as well as the increased susceptibility to Hodgkin's disease among individuals with HL-A antigens of the $4 \mathrm{C}$ system reported by Falk and Osoba. ${ }^{13}$ As in other malignancies of the lymphoid system the cause may prove to be multifactorial-an interaction of environmental and genetic factors.

The present debate had largely been foreshadowed by the earlier demonstration of a bimodal age incidence by MacMahon. ${ }^{14}$ In the north-eastern U.S.A. and most northern European countries there is an early peak between 15 and 35 years of age and a later peak over the age of 50 . In southern 
states of the U.S.A. and in Japan this early peak is lacking. MacMahon suggested that Hodgkin's disease was an infectious condition in the young and a neoplasm in the old. The bimodality could equally be explained if older people acquired the disease from their children, bearing in mind the postulated long incubation period. ${ }^{15}$ Another recent development has been the appearance of an early peak among Japanese living in the U.S.A. Mason and Fraumeni ${ }^{16}$ have interpreted this shift in the American-Japanese curve towards that of the white Americans as consistent with an environmental influence affecting all ages rather than with an infective agent affecting only young adults. Clarke, Anderson, and Davidson ${ }^{17}$ have recently presented an alternative working hypothesis in which a transmissable form of Hodgkin's disease may occur at any age.

Where, then, do we stand at present ? On current evidence it is premature to conclude that case-to-case transmission of Hodgkin's disease occurs in the school, surgery, or family home. Furthermore, the postulated increase in incidence in these situations is only of the order of two-to-eightfold, which in practical terms represents a negligible hazard in a disease with an overall incidence of around 4 per 100000 population per year. Doctors, teachers, spouses, and close relatives of patients with this disease may take heart from these facts.

\footnotetext{
1 Vianna, N. J., Greenwald, P., and Davies, J. N. P., Lancet, 1971, 1, 1209.

2 Vianna, N. J., et al., Annals of Internal Medicine, 1972, 77, 169.

3 Klinger, R. J., and Minton, J. P., Lancet, 1973, 1, 168.

4 Smith, P. G., Pike, M. C., and Kinlen, L. J., Lancet, 1973, 1, 433.

5 Vianna, N. J., and Polan, A. K., New England fournal of Medicine, 1973, $289,499$.

6 Milham, S., New England fournal of Medicine, 1974, 290, 1329.

7 Bahn, A. K., New England fournal of Medicine, 1974, 291, 207

${ }^{8}$ Hoover, R., New England fournal of Medicine, 1974, 291, 473.

9 Vianna, N. J., et al., Lancet, 1974, 2, 131.

10 Smith, P. G., Kinlen, L. J., and Doll, R., Lancet, 1974, 2, 525.

11 Vianna, N. J., et al., Lancet, 1974b, 2, 854

12 MacMahon, B., Cancer Research, 1966, 26, 1189.

13 Falk, J., and Osoba, D., Lancet, 1971, 2, 1118.

14 MacMahon, B., Cancer, 1957, 10, 1045.

15 Wagener, D. J., Lancet, 1974, 1, 880.

16 Mason, T. J. and Fraumeni, J. F Lancet, 1974, $1,745$.

17 Clarke, E. A., Anderson, T. W., and Davidson, J. W., Lancet, 1974, 1, 745.
}

\section{Transient Ischaemic Attacks}

Transient ischaemic attacks (T.I.A.s) are a common presenting symptom of cerebrovascular disease. The characteristic clinical picture is one of a focal neurological deficit of abrupt onset, lasting up to 24 hours, and leaving no residual signs. In about $30 \%$ of cases a completed stroke occurs after one or more T.I.A.s, usually within 3 years, ${ }^{12}$ but the prognosis is rather better in attacks arising in the vertebrobasilar artery territory. ${ }^{3}$

Most T.I.A.s are due to the passage of an embolus through the cerebral circulation. More rarely they are caused by reduction of perfusion through a grossly narrowed vessel-the "haemodynamic crisis" of Denny-Brown-secondary to sudden blood loss, hypotension, or a fall in cardiac output.

T.I.A.s are characterized by their sudden onset, often in waking hours, and by the repetitive nature of the symptoms in an individual-they may recur several times a day. As has recently been shown by Grindal and Toole, ${ }^{4}$ headache may be prominent in up to $25 \%$ of patients during or after an attack. Its location is variable, but in vertebrobasilar attacks it is often in the neck or occiput, while pain is more typically frontal or temporal in carotid attacks. An earlier report ${ }^{5}$ noted a higher incidence of headache in non-dominant hemisphere lesions and considered that dysphasia might mask it when the speech was affected in lesions of the left hemisphere. The mechanism of the headache is uncertain, but it may be related to distension of pain-sensitive nerve fibres in the arterial wall at the site of impaction of the embolus; dilatation of collateral vessels has also been suggested.

In the carotid territory the common symptoms are unilateral weakness of the face, arm, or leg, with or without speech disturbance. Hemianopic field defects occur in posterior cerebral artery occlusion and also (in association with hemianaesthesia) in ischaemic lesions of the internal capsule. Transient monocular blindness (amaurosis fugax) ${ }^{6}$ may afford the diligent observer the chance of seeing bright yellow cholesterol crystals or whitish fibrin-platelet plugs traverse the retinal vessels. More often the history of a "curtain" or "shutter" crossing the visual field points unmistakably to a source of embolus in the carotid or great vessels, including the aorta and the heart itself.

A bruit localized over the carotid artery is a good clue to a stenosis or even an occlusion; a similar bruit in the supraclavicular fossa or the posterior triangle of the neck suggests a subclavian or vertebral stenosis. Usually this is accompanied by a diminished radial pulse and a drop of blood pressure of over $20 \mathrm{~mm} \mathrm{Hg}$ in the arm. The common symptoms of vertebrobasilar attacks are vertigo, diplopia, fortification spectra, hemianopia, and paraesthesiae especially around the lips and face; there may also be ataxia, slurred speech, and numbness of both arms or legs, or of one side. Hemiparesis and hemisensory disturbance seem to carry a greater risk of a forthcoming infarct. A variant of this picture is the "subclavian steal syndrome," in which symptoms are precipitated by exercise of the arm, which increases the demand for blood. The narrowed subclavian artery then "steals" blood from the vertebral artery. The reversed flow can be seen at angiography and can be treated by endarterectomy-if symptoms are disabling.

Investigation of carotid T.I.A.s is designed to find treatable factors and thus prevent a disabling stroke. Age is a poor guide, and most reasonably fit patients merit assessment in a neurological unit. Tests will include a full blood count, E.S.R., radiographs of chest and skull, electrocardiogram, and fasting blood glucose, lipids, and urea. Exhaustive routines are not justifiable-an individual assessment of each case should determine the need for further investigation. Evidence of widespread coronary and peripheral vascular disease will often contraindicate angiography; so too will sustained hypertension, unless a localized neck bruit suggests a surgically remediable stenosis.

With these exceptions angiography is usually indicated in patients with carotid T.I.A.s. The morbidity is less than $0.5 \%$ and the mortality almost negligible in skilled hands. The aim is to show the extent and number of vascular stenoses or occlusions (multiple or clinically unsuspected lesions being common) and to exclude lesions such as meningiomas or angiomas which may mimic a stroke.

In patients with vertebrobasilar T.I.A.s the incidence of surgically correctable lesions is small. Only those cases with severe symptoms related to neck movement suggesting vertebral compression by removable cervical osteophytes or those with subclavian steal syndrome should be considered for surgery, and hence for angiography.

Before contemplating surgery, an arch aortogram showing all four major neck vessels is necessary: initial enthusiasm may be dampened by the multiple lesions often seen. In an isolated carotid stenosis operation carries a better outlook than anticoagulants or doing nothing. " Thromboendarterectomy carries a mortality rate $^{8}$ of about $1 \%$, and postoperative 